\title{
Polymorphous Low-Grade Adenocarcinoma in the Nasopharynx: A Case Report and Review of the Literature
}

\author{
Tae Ui Hong, Seong Kook Park (D), Do Hun Kim, and Sihong Choe \\ Department of Otorhinolaryngology-Head and Neck Surgery, College of Medicine, Inje University, Busan Paik Hospital, Busan, Korea
}

비인두에서 발생한 다형성 저등급 선암종 1예

홍태의 · 박성국 · 김도훈 · 최시홍

인제대학교 의과대학 부산백병원 이비인후-두경부외과학교실

\author{
Received December 30, 2019 \\ Revised March 16, 2020 \\ Accepted March 17, 2020 \\ Address for correspondence \\ Seong Kook Park, MD, PhD \\ Department of Otorhinolaryngology- \\ Head and Neck Surgery, \\ College of Medicine, \\ Inje University, Busan Paik Hospital, \\ 75 Bokji-ro, Busanjin-gu, \\ Busan 47392, Korea \\ Tel $+82-51-890-6379$ \\ Fax $+82-51-892-3831$ \\ E-mail sinus4@paik.ac.kr
}

Polymorphous low-grade adenocarcinoma (PLGA) is a neoplasm that usually occurs in the minor salivary glands located in the mucosa of the soft and hard palates, the buccal mucosa, and the upper lip. It rarely occurs in the nasopharynx, and only six cases have been reported. PLGA can be treated by wide local excision and requires no additional treatment if a negative margin is identified in frozen biopsy. Here we describe a case of PLGA originating in the nasopharyngeal mucosa. We were able to completely resect the tumor using simple endoscopic surgery rather than using an open approach, such as a transpalatal resection. Our patient had no local recurrence or distant metastasis at the 24-month follow-up.

Korean J Otorhinolaryngol-Head Neck Surg 2021;64(2):119-23

Key Words Endoscopic surgery · Minor salivary gland · Nasopharynx

Polymorphous low-grade adenocarcinoma.

\section{Introduction}

Polymorphous low-grade adenocarcinoma (PLGA) is a minor salivary gland neoplasm that primarily occurs in intraoral sites. An extraoral location of the tumor is rare, with nasal cavity tumors representing $<1 \%$ of PLGAs. ${ }^{1)}$ The tumor generally involves the palate but has been reported to occur in the base of the tongue, upper lip, buccal mucosa, tonsils, and retromolar pad. ${ }^{2)}$ PLGA usually occurs in the fifth and sixth decades of life with a female/male ratio of $2: 10^{3,4)}$

To date, only six cases of PLGA presenting as a nasopharyngeal mass have been reported; none have been reported in Korea. ${ }^{5-10)}$ Nasopharyngeal PLGA grows slowly, painlessly, and has few symptoms, including unilateral ear fullness and

This is an Open Access article distributed under the terms of the Creative Commons Attribution Non-Commercial License (https://creativecommons.org/licenses/by-nc/4.0) which permits unrestricted non-commercial use, distribution, and reproduction in any medium, provided the original work is properly cited. nasal obstruction. Here, we describe a case of nasopharyngeal PLGA including clinical characteristics, pathology, treatment, and prognosis, along with a review of the literature.

\section{Case}

A 51-year-old male patient presented with right-side ear fullness without nasal symptoms. No neck mass was noted. He did not smoke or drink alcohol and had no past history of acute disease, with the exception of cataract surgery. Nasal endoscopy revealed an irregularly shaped mass on the right salpingopalatine fold (Fig. 1). Paranasal sinus CT showed a $20 \times 23 \times 30$-mm polypoid mass in the lateral wall of the right nasopharynx without surrounding bony destruction or intraorbital or skull base invasion (Fig. 2). A right middle ear effusion was observed on otoscopy. Punch biopsy of the nasopharyngeal mass was performed in the outpatient department. 

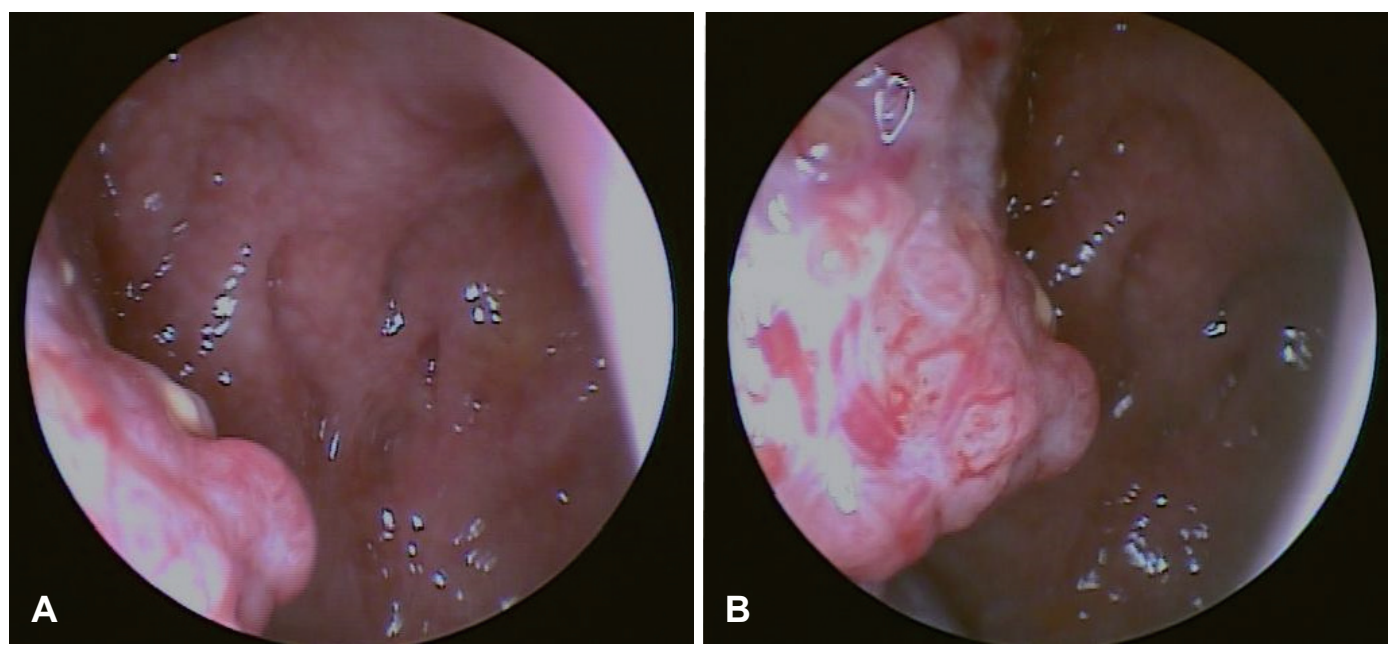

Fig. 1. Transnasal endoscopic findings. The tumor is located in the right salpingopalatine fold (A). The right Eustachian tube appears to be blocked $(B)$.
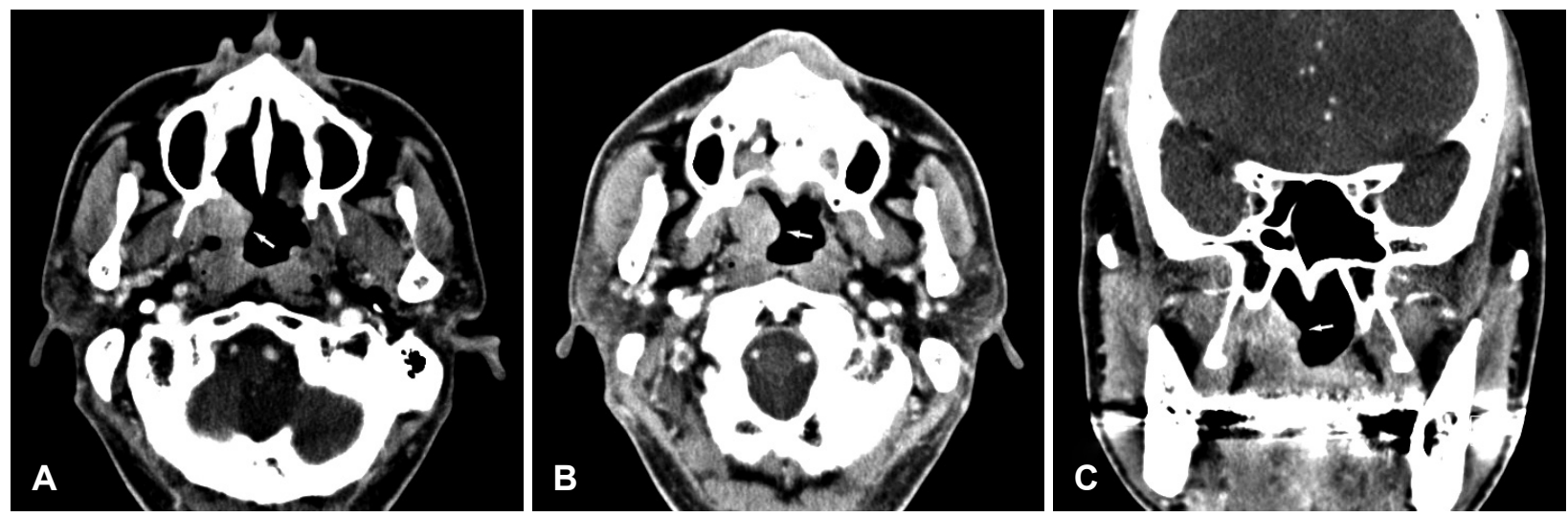

Fig. 2. Paranasal sinus CT scan shows a well-defined, heterogeneous mass $(20 \times 23 \times 30 \mathrm{~mm})$ (arrow) originating from the right nasopharyngeal lateral wall mucosa $(\mathrm{A}-\mathrm{C})$. The surrounding bony structure is unaffected.
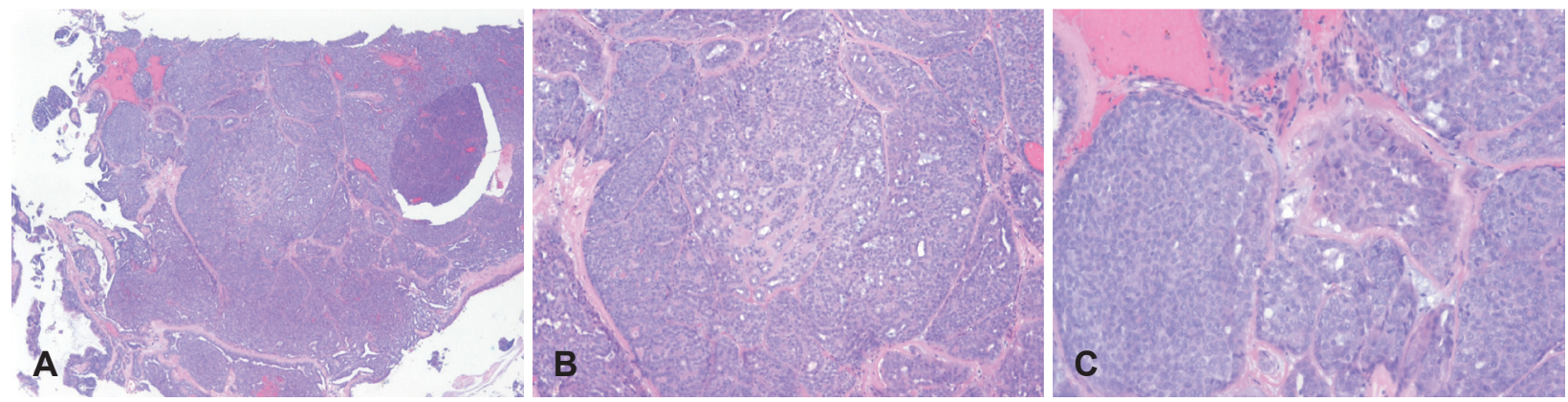

Fig. 3. Histologic findings of the specimen obtained by punch biopsy performed in the outpatient department. Monotonous epithelial cells are arranged in well-marginated nests and along the tumor nests. Small hyperchromatic nuclei and spindle-shaped myoepithelial cells are present between and in the tumor cell nests; focal myxoid stromal matrix is observed. These findings are compatible with a pleomorphic adenoma. Hematoxylin and eosin stain: A $(\times 40), B(\times 100)$, and C $(\times 200)$.

Pathology revealed a benign salivary gland tumor consistent with pleomorphic adenoma (Fig. 3).

The tumor was resected using an endoscopic approach, without further evaluation because the preoperative biopsy result was definitively benign. ${ }^{11)}$ Under general anesthesia, the transnasal endoscopic approach was used with 0 - and 30-degree 4-mm nasal endoscopes (Karl Storz SE \& Co. KG, Tuttlingen, Germany). The posterior portion of the right inferior turbinate was excised to create a working space for the endoscope and to expose the tumor. A circular incision of the mucosa was then made around the mass using a sickle knife, and further dissection was performed using a Freer el- 

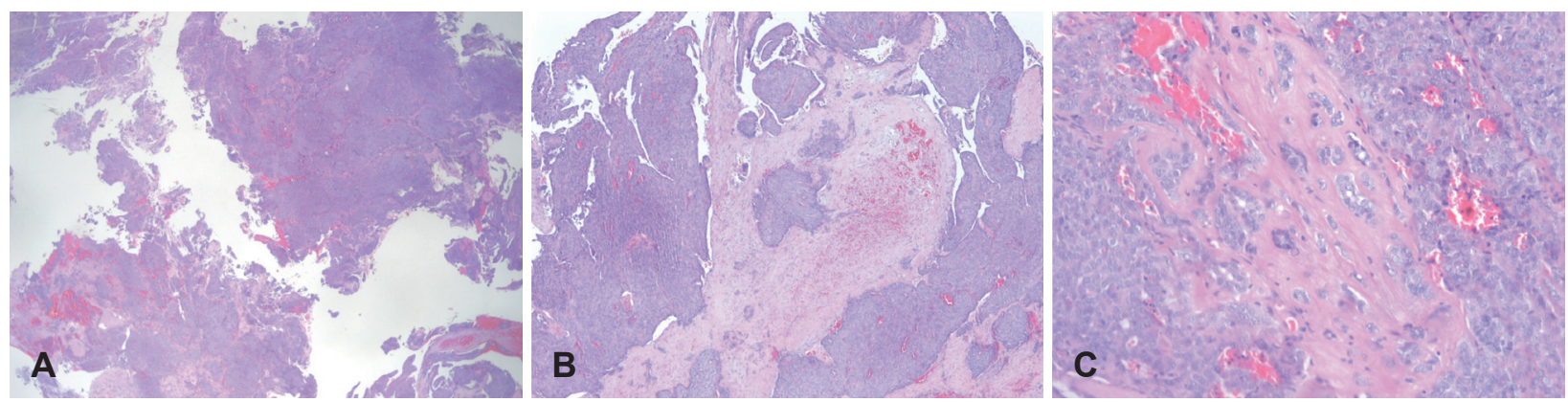

Fig. 4. Histopathologic findings of PLGA involving the nasopharynx. Hypercellular and compact tumor cells are arranged in irregularly shaped nests $(A: H \& E$ stain, $\times 10)$. Multiple tumor cell clusters are scattered in the pinkish stromal matrix between tumor nests, suggestive of an invasive front (B: H\&E stain, $\times 40)$. Multifocal hemorrhagic foci are visible in the tumor cell nests (C: H\&E stain, $\times 100)$. H\&E: hematoxylin and eosin.
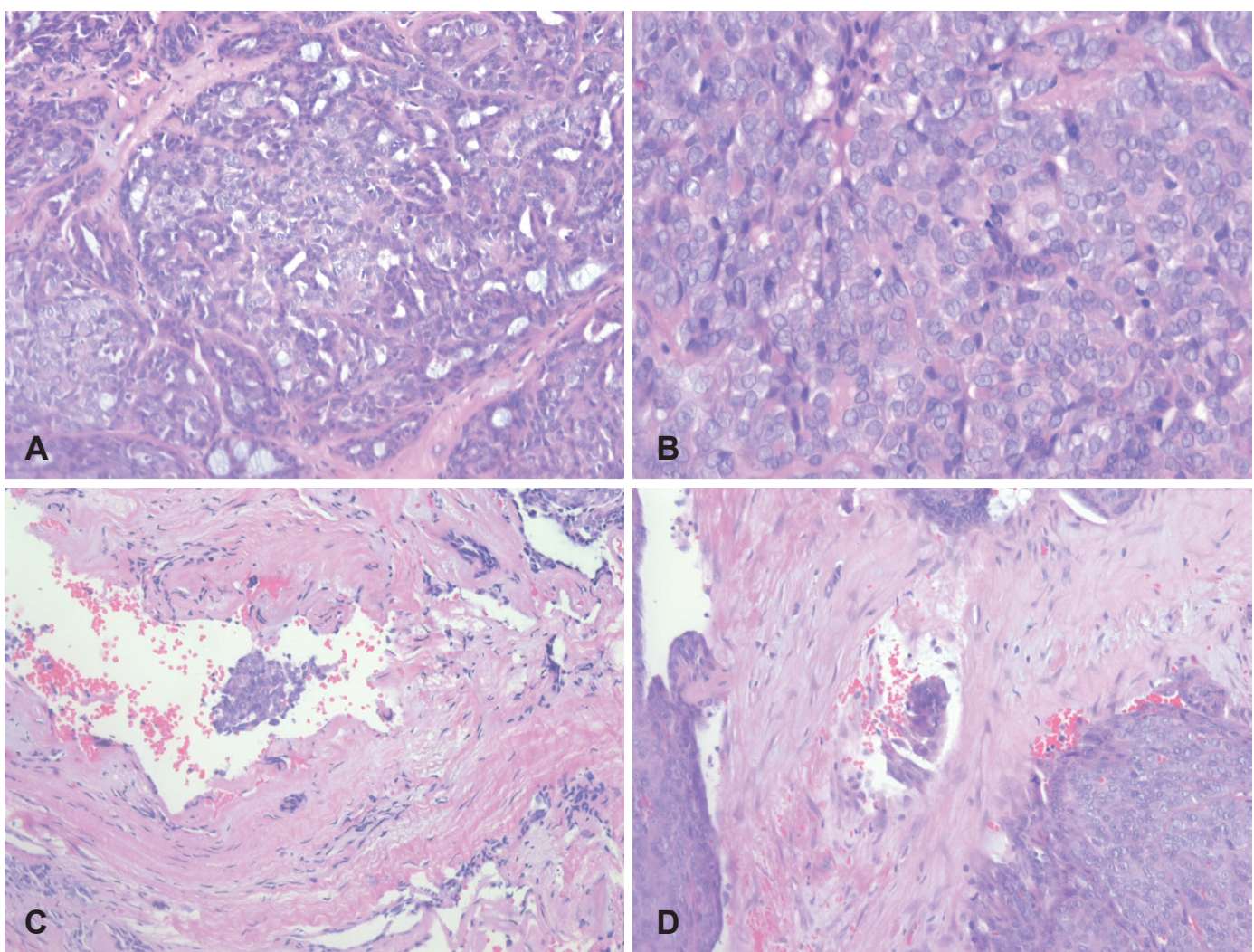

Fig. 5. H\&E stain results at higher magnification. Tumor cells arranged in papillary and cribriform patterns are observed (A: H\&E stain, $\times 200$ ). Mitotic figures are rare but present. Small- to medium-sized tumor cells with mildly hyperchromatic and oval nuclei are observed (B: H\&E stain, $\times 400)$. Two foci of lymphovascular invasion are observed (C, D: H\&E stain, $\times 400)$. H\&E: hematoxylin and eosin.

evator. The right pterygoid bone was exposed after the tumor was excised with a 2-mm resection margin. Frozen biopsy specimens revealed a carcinoma of undetermined type. Frozen sections prepared from the intraoperative margin tissue revealed a negative resection margin. The gross surgical specimen showed a pale brown to tan soft tissue mass measuring $20 \times 13 \times 10 \mathrm{~mm}$; it was reduced in size compared to the preoperative $\mathrm{CT}$, due to the outpatient punch biopsy. Finally, a fibrin-collagen patch (TachoComb ${ }^{\circledR}$; Nycomed, Linz, Austria) was applied to the wound site, followed by Merocel ${ }^{\circledR}$
(Medtronic, Jacksonville, FL, USA) packing from the right posterior nasal cavity to the nasopharyngeal cavity. The Merocel was removed 2 days post-surgery; the patient was discharged from the hospital on the following day without significant complications. The final diagnosis was PLGA with the resection margin free of tumor (Figs. 4-6). A PET-CT scan confirmed no remnant nasopharyngeal mass or neck metastasis. Follow-up PET-CT performed at 7 months after nasal endoscopic surgery revealed no abnormal lesion suggestive of recurrence. The right middle ear effusion subsequently 

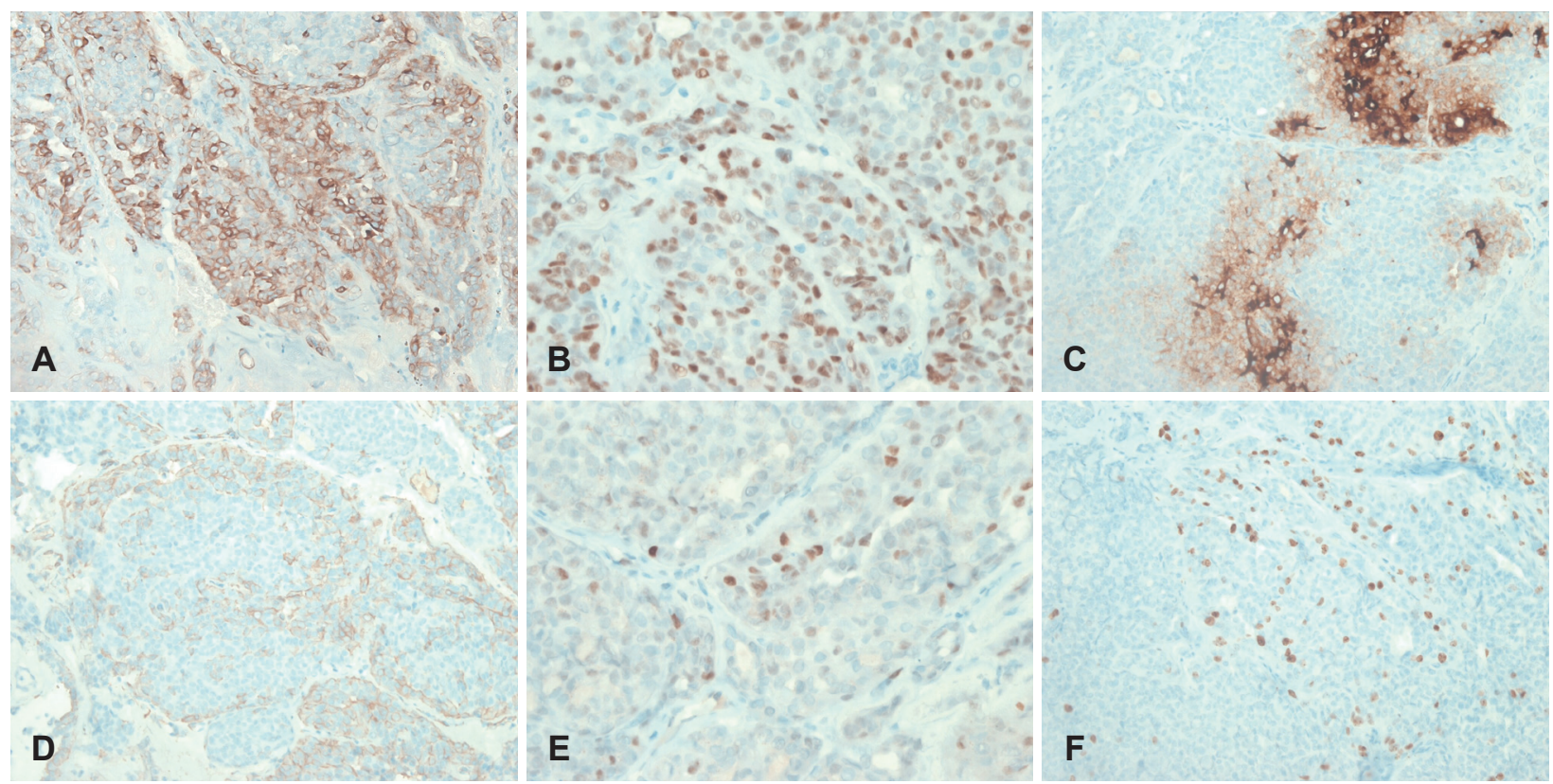

Fig. 6. Immunohistochemical findings. The tumor cells were positive for $C K(\times 200)(A)$ and p63 $(\times 400)(B)$; focal positive for EMA $(\times 200)(C)$, SMA $(\times 200)(D)$, and p53 $(\times 400)(E)$; and 20\% positive for Ki-67 $(\times 200)(F)$. CK: cytokeratins, EMA: epithelial membrane antigen, SMA: smooth muscle antibody.
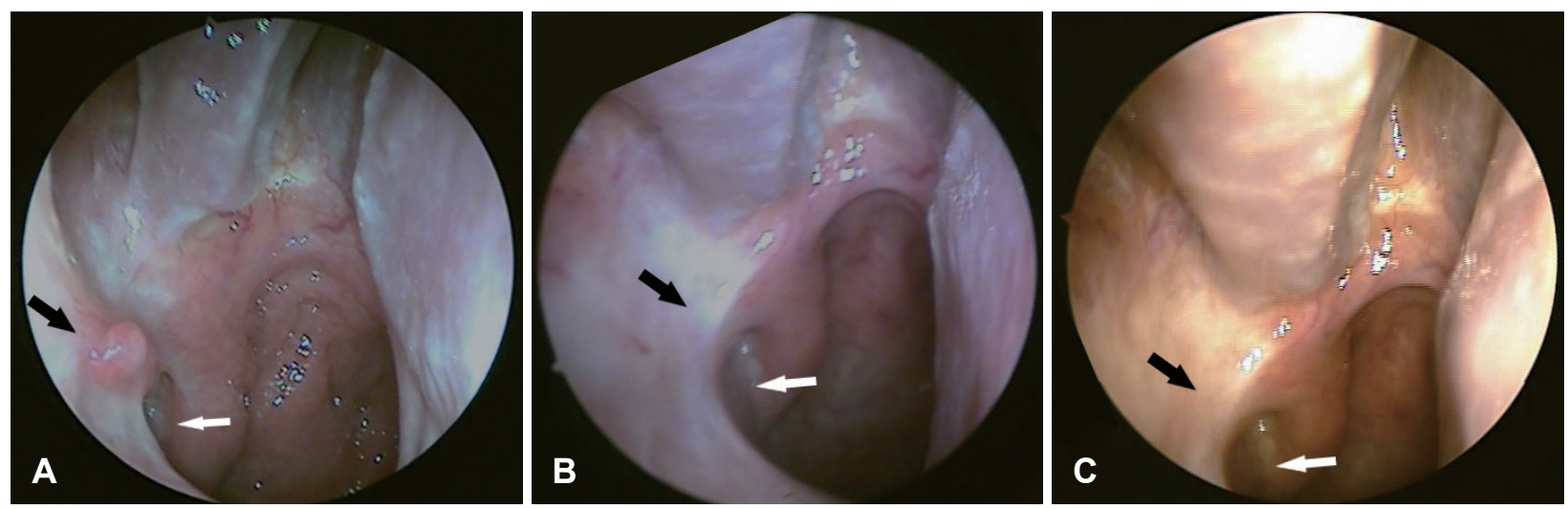

Fig. 7. Endoscopic findings at 3 months (A), 12 months (B), and 24 months (C) show a clear surgical site (black arrow) and no recurrence after transnasal endoscopic surgery. The Eustachian tube orifice is clearly visible (white arrow).

improved and is undergoing follow-up monitoring. At the 24-month follow-up, the patient showed no signs of local recurrence or distant metastasis (Fig. 7).

\section{Discussion}

Histologically, PLGA is characterized by its morphologic variability, cytologic blandness, and an infiltrative growth pattern. ${ }^{5)}$ In most instances, the tumors are grossly circumscribed; however, they are unencapsulated and invade adjacent tissues microscopically. Neurotropism is a frequent finding. The growth pattern varies and includes solid islands, tubules, trabeculae, cribriform nests, cysts, and papillae. The lesions that most frequently cause diagnostic difficulties with
PLGA are pleomorphic adenoma and adenoid cystic carcinoma. ${ }^{5)}$ PLGA is locally infiltrative, often with prominent perivascular and perineural invasion. These features can help to differentiate this tumor from both pleomorphic and monomorphic adenomas; usually, well-circumscribed tumors lack the infiltrative nature of PLGA. Differential diagnosis of PLGA from adenoid cystic carcinoma is difficult. Many of the histologic features of these two entities (solid and cribriform patterns and neurotropism) are similar; histologic distinction is virtually impossible. Immunohistochemical findings can distinguish the two lesions; PLGA exhibits positive EMA, S-100, and CEA findings.

Clinically, PLGA has a low tendency for aggressive behavior; thus, the recommended treatment is surgical excision, 
and the results are generally excellent. No cases of lymphatic spread have been described; therefore, neck dissection is unnecessary unless there is clinical evidence of lymph node involvement. If the tumor has not been completely resected, adjuvant radiotherapy should be performed to prevent tumor recurrence. ${ }^{6)}$ PLGA rarely occurs in the nasopharynx, and there is no definite treatment of choice. En-bloc mass excision using the transpalatal or nasal endoscopic approaches are options; however, the transpalatal approach may cause several complications, including pain, bleeding, and oronasal fistulas. Exclusive endoscopic resection can avoid potential complications and shorten the hospital stay.

To date, six cases of nasopharynx PLGAs have been reported. Lengyel, et al. ${ }^{7)}$ reported a case with skull base invasion and intracranial spread confirmed by CT. Radiotherapy was performed. Follow-up CT scans revealed a partial response to radiotherapy, and the patient was still living at 51 months postradiotherapy. Wenig, et al. ${ }^{5)}$ reported a patient with PLGA who underwent transpalatal tumor resection and exhibited uneventful recovery at 9 months after the operation. Furthermore, four previous studies found that patients with PLGA who underwent exclusive transnasal endoscopic tumor resection recovered fully without additional treatment. ${ }^{6,8-10)}$ Our case is the fifth report of a patient with nasopharyngeal PLGA who underwent nasal endoscopic tumor resection without additional therapy. Our findings, together with those of previous studies, suggest that the simple endoscopic approach is a feasible treatment for nasopharyngeal PLGA. However, during endoscopic surgery, the surgeon must be able to resect the mass with adequate safety margins to ensure they are free of tumor (e.g., by identifying negative margins in frozen biopsy). When the tumor extends beyond the nasopharynx or endoscopic view, we suggest that open surgery (e.g., transpalatal approach) should be used, rather than the endoscopic approach. In addition, open surgery should be performed when local recurrence is detected after endoscopic surgery.

In patients with nasopharyngeal PLGA limited to the nasopharyngeal mucosa, the endoscopic approach is feasible if the surgeon is able to resect the PLGA with sufficient margins. This procedure is curative, can reduce the length of the operation, decrease the incidence of wound-related complications, and shorten the hospital stay.

\section{Acknowledgments}

None.

\section{Author Contribution}

Conceptualization: Seong Kook Park. Data curation: Tae Ui Hong, Sihong Choe. Formal analysis: Tae Ui Hong, Seong Kook Park. Methodology: Tae Ui Hong, Seong Kook Park, Do Hun Kim. Project administration: Do Hun Kim. Supervision: Seong Kook Park. Writing - original draft: Tae Ui Hong, Sihong Choe. Writing - review \& editing: Seong Kook Park, Do Hun Kim.

\section{ORCID}

Seong Kook Park https://orcid.org/0000-0003-3903-8412

\section{REFERENCES}

1) González-Lagunas J, Alasà-Caparrós C, Vendrell-Escofet G, Huguet-Redecilla P, Raspall-Martin G. Polymorphous low-grade adenocarcinoma of the nasal fossa. Med Oral Patol Oral Cir Bucal 2005;10(4):367-70.

2) Luna MA, Batsakis JG, Ordóñez NG, Mackay B, Tortoledo ME. Salivary gland adenocarcinomas: A clinicopathologic analysis of three distinctive types. Semin Diagn Pathol 1987;4(2):117-35.

3) Charous DD, Cunnane MF, Rosen MR, Keane WM. Recurrent polymorphous low-grade adenocarcinoma manifesting as a sinonasal mass: A case report. Ear Nose Throat J 2005;84(6):354, 356-7.

4) Clayton JR, Pogrel MA, Regezi JA. Simultaneous multifocal polymorphous low-grade adenocarcinoma. Report of two cases. Oral Surg Oral Med Oral Pathol Oral Radiol Endod 1995;80(1):71-7.

5) Wenig BM, Harpaz N, DelBridge C. Polymorphous low-grade adenocarcinoma of seromucous glands of the nasopharynx. A report of a case and a discussion of the morphologic and immunohistochemical features. Am J Clin Pathol 1989;92(1):104-9.

6) Lai YS, Chen MK. Exclusively endoscopic resection of nasopharyngeal adenocarcinoma. Clin Exp Otorhinolaryngol 2013;6(4):263-5.

7) Lengyel E, Somogyi A, Gõdény M, Szerdahelyi A, Németh G. Polymorphous low-grade adenocarcinoma of the nasopharynx. Case report and review of the literature. Strahlenther Onkol 2000; 176(1):40-2.

8) Wei YC, Huang CC, Chien CY, Hwang JC, Chen WJ. Polymorphous low-grade adenocarcinoma of the nasopharynx: A case report and brief review. J Clin Pathol 2008;61(10):1124-6.

9) Garzaro M, Pecorari G, Landolfo V, Campisi P, Reali A, Giordano C. Nasopharyngeal polymorphous low-grade adenocarcinoma in a patient with nonfunctioning pituitary macroadenoma. B-ENT 2010;6(1):59-62.

10) Kaul R, Gulati A, Kaushik R, Raina S. Polymorphous low grade adenocarcinoma-an unusual presentation. Online J Health Allied Scs 2010;9(2):18.

11) Martínez-Capoccioni G, Martín-Martín C, Espinosa-Restrepo F. Transnasal endoscopic resection of a nasopharyngeal pleomorphic adenoma: A rare case report. Eur Arch Otorhinolaryngol 2012;2 69(8):2009-13. 